\title{
Nutritional support in malignant disease
}

\section{By K. C. Calman, Department of Clinical Oncology, Gartnavel General Hospital, Glasgow G12 $2 L Y$}

The patient with cancer has multiple metabolic and nutritional abnormalities. The indications and methods of nutritional support required may therefore vary considerably from patient to patient. Currently many techniques are available for the administration of nutritional support. Rather than review each of these in detail it is considered more appropriate to pose specific questions in relation to nutrition and cancer.

\section{Nutritional problems of cancer patients}

In any heterogeneous group of patients with a variety of cancers, the nutritional abnormalities reported may be very different indeed. In some cases the patient may be entirely normal, and, even when the disease is metastatic, the patient may show no clinical or laboratory evidence of nutritional deficiencies. In advanced disease, however, it is usual to find some evidence of nutritional abnormalities. In a recent nutritional survey of 120 patients with cancer referred to the clinic the abnormalities shown in Table I were recorded (M. Soukop, R. A. McAllister, R. A. Fleck, J. G. McVie \& K. C. Calman, unpublished observations). Clinically such patients showed evidence of weight loss, loss of skinfold fat and felt weak and tired. These signs and symptoms, in association with muscle wasting and an increased basal metabolic rate constitute the syndrome of cachexia. The aetiology of this condition which is complex has recently been reviewed (Theoglides, 1972). Anorexia is another major symptom in cancer patients and this may be extremely difficult to treat. Taste abnormalities are also common and may be associated with trace-metal deficiencies (DeWys \& Walters, I975). In addition to compounding the clinical syndrome the low serum-protein levels noted may alter drug binding and influence toxicity. Vitamin deficiencies are very common. Of particular clinical interest at present are vitamin $C$ and vitamin $A$. The importance of these will be discussed later. Trace-metal abnormalities are also common (Pories, Mansour \& Strain, 1972; Mortazavi, Bani-Hashemi, Mozafari \& Raffie, 1972) and they too may be relevant when therapy is considered.

The mechanisms of such abnormalities still remain unresolved. In some patients the answer is simply due to diminished food intake, or mechanical obstructive problems. In others this cannot be the whole answer. The evidence that tumours release products which alter metabolic processes is still not completely convincing though the hypothesis remains of considerable interest (Okuda, Ikegami \& Fujii, 1972; Goodlad \& Raymond, 1973). 


\section{Methods of nutritional support}

Methods available include oral techniques such as supplemental feeding, elemental nutrition, and tube feeding. Intravenous feeding may be total or supplemental. As anorexia is a special problem in cancer patients it is worth considering the use of anabolic agents and appetite stimulants. Few good clinical trials have documented evidence that such agents as the anabolic steroids, prednisolone or other appetite stimulants are of value. Psychological support and the judicious use of alcohol may be as effective.

\section{Indications for nutritional support}

The nutritional problems having been documented, and the methods of support currently available briefly described, it is possible to consider a large number of potential indications for nutritional support in patients with cancer. Nutritional support may be employed before commencement of therapy, once the diagnosis is made. One of the most common problems envisaged is the patient with head and neck, oesophageal or gastric cancer about to undergo radiotherapy or surgery. In such patients parenteral nutrition has been widely used before therapy in the hope that wound healing would be improved and post-treatment complications would be diminished.

The second group of indications is the use of nutritional support during therapy. Thus patients with carcinoma of the oesophagus given radiotherapy may temporarily obstruct, and intravenous nutrition may be necessary. Intensive chemotherapy, given over a period of days may result in a substantial loss of bodyweight. Recently, a number of studies have used parenteral nutrition to support patients during such treatments and these will be reviewed later.

The next group of indications is in the post-treatment patient, where support may be used in one of two ways; either routinely where a problem is anticipated, or, when a complication occurs. In the former it might be used following operations in the head and neck regions or on the gastrointestinal tract, when a period of oral food intake is not considered possible. When complications of surgery, radiotherapy or chemotherapy occur nutritional support may be necessary. Such indications would include poor wound healing, the development of sinues, fistulae and anastomotic leaks.

The final group of indications is in the treatment of advanced disease. In this case the psychological problems of such patients may make it important that they eat as normally as possible. Nutritional supplementation and appetite stimulants may be used in an attempt to prevent weight loss.

\section{The assessment of results}

In the past many nutritional studies have been conducted in cancer patients without adequate attempts being made to assess results; no definite end-point has been used and patients have been recorded simply as being better or worse. The reason, of course, is that nutritional assessment is extremely difficult. Soukop et al. 
(unpublished results) show that there is a good correlation between anthropometric measurements, biochemical results, and the clinical condition of the patient. Whenever possible a reliably measurable parameter should be used to assess results. In cancer patients, it is also essential that the tumour status is accurately recorded and an attempt made to quantitate the tumour mass. The progress or regression of the tumour should be recorded throughout the study. This is crucial, as recently suggestions have been made as to the potential harm which may result from nutritional support (Burke \& Kark, 1977).

\section{The usefulness of nutritional support}

For very many years now there have been innumerable studies on this subject and it would be impossible to review them all. Reference will only be made, therefore, to those studies which have shown (a) that nutritional support maintained the patient nutritionally, or showed improved results; (b) prevented the development of treatment side effects; or (c) enhanced response rates of the tumour. In this respect it is important to distinguish between those studies in which nutritional support alone was given, and those in which concomitant antitumour therapy was also administered. No reference will be made to wound healing or post-operative complications.

Intravenous hyperalimentation. A number of studies have now shown that intravenous hyperalimentation may prevent some of the nutritional consequences of treatment. During radiotherapy for example (Copeland, Souchon, MacFadyen, Rapp \& Dudrick, 1977) there was a significant weight gain during therapy in patients who were nutritionally depleted. There was also a positive correlation between the tumour response and nutritional status. In this study objective tumour responses occurred in $54 \%$ of patients, indicating that the nutritional support had not abolished the tumour response. No control group without hyperalimentation was recorded, however.

Increasing use is being made of intensive chemotherapy in some forms of neoplasm. This is particularly the case in the testicular teratomas where combinations of vinblastine and bleomycin are used. In a recent report, Samuels and colleagues (Samuels, Lanzotti, Hologe, Boyle, Smith \& Johnson, 1976) have shown that in twenty-four patients given intravenous hyperalimentation the mean weight gained during courses was $0.45 \mathrm{~kg}$. When patients were not given hyperalimentation the mean weight loss was $4.95 \mathrm{~kg}$. Personal clinical experience in the use of these drugs in the teratomas provide convincing evidence of the weight loss suffered by these patients and the protective effect of nutritional support.

A further study (Lanzotti, Copeland, George, Dudrick \& Samuels, 1975) of considerable importance indicates that the response rate to chemotherapy may be improved in patients who are nutritionally depleted. In an uncontrolled study of patients with lung cancer it was shown that patients who were nutritionally abnormal, and in whom low response rates to chemotherapy were characteristic, could be made to respond more frequently following nutritional support. Urgent confirmation of these findings is necessary. 
In summary, several studies have indicated that intravenous hyperalimentation may be beneficial but that controlled results are urgently required. There is no indication, at the present time, of adverse effects of this method of therapy in relation to tumour growth.

Elemental diets. The role of elemental diets in the cancer patient have recently been reviewed (Calman, 1976). Such diets have specific indications in patients who have evidence of malabsorption, intestinal obstruction or fistulae. They may be of considerable value in patients who have dysphagia. Of particular interest in the cancer area is the report (Bounous, Gentile \& Hugon, 1971) that elemental diets could protect the small and large intestinal mucosa against chemotherapeutic agents, notably 5 -Fluorouracil. Several studies are at present underway to confirm or refute this report.

Nutritional supplementation. A wide variety of oral preparations are now available. They are used in many clinical situations, but there is little evidence in the cancer patient that their use is associated with benefit or harm. Adequate controlled studies are required.

Oral force feeding or tube feeding. There is conflicting evidence on the use of these methods in cancer patients. Pareira, Conrad, Hicks \& Elman (1955) suggest that it may be beneficial in that some patients may gain weight and begin to eat normally, on the other hand, Terepka \& Waterhouse (1956) have indicated that there may be acceleration of the disease. This, however, was a small study with only six patients. It is important, however, to realize that such studies were performed without the use of concomitant anti-cancer therapy. This is in contrast to the results previously detailed with hyperalimentation.

\section{Supplementation with vitamins and trace metals}

From the results presented in Table $I$ it is clear that many patients with advanced disease are vitamin deficient, and this has been confirmed many times (Tannenbaum \& Silverstone, 1953). Similarly many patients are given vitamin supplementation but few studies have clearly documented the value of this. The majority of the information is related to vitamin $C$, vitamin $A$, and riboflavin. The role of folic acid, its antagonists and derivatives in cancer therapy will not be discussed here. Thiamine deficiencies are common and this may be clinically relevant in relationship to drug metabolism (Basu, Dickerson, Raven \& Williams, 1974).

Ascorbic acid. This vitamin has multiple functions in cancer patients. It may be associated with the development of malignant disease (Stitch, Karim, Koropatnick \& Lo, 1976) and with its treatment (Cameron \& Pauling, 1976). High-dose ascorbic acid has been used in the treatment of patients with advanced cancer and there is some evidence that patients may live longer, given this form of treatment. More important, perhaps, is that ascorbic acid may be essential for drug detoxication (Zannoni \& Rikans, 1976) and may therefore modify the activity of chemotherapeutic agents. 
Table 1. Nutritional abnormalities in cancer patients, survey of 120 patients

$\begin{array}{cr}\text { Anaemia } & \% \\ \text { Vitamin A deficiency } & 49 \\ \text { Carotene deficiency } & 15 \\ \text { Thiamine deficiency } & 15 \\ \text { Abnormal leucocyte ascorbic acid } & 37 \\ \text { Folate abnormal } & 71 \\ \text { Vitamin } B_{12} \text { abnormal } & 23 \\ \text { Low serum albumin } 35 \mathrm{~g} / 1 & 0 \\ \text { Weight loss o-6\% 30 g/1 } & 47 \\ 7-15 \% & 20 \\ 16-25 \% & 24 \\ 20+ & 31 \\ \text { Skin-fold thickness normal } & 31 \\ \text { lean } & 14 \\ \text { low } & 22 \\ \text { very low } & 33 \\ & 15 \\ \end{array}$

Vitamin $A$. This vitamin is of particular interest in patients with cancer. Not only is it associated with carcinogenesis (Newberne \& Rogers, 1973), but it has been advocated as a form of propylaxis against cancer (Anonymous, 1976) and virus infection (Seifter, Rettura, Demetrion \& Levenson, 1976). It acts as an immunological adjuvant (Jurin \& Tannock, 1972; Meltzer \& Cohen, 1974), potentiates chemotherapeutic agents in small animal tumour models (Sydner, Allen \& Flesher, 1972; Cohen \& Carbone, 1972) and humans (Prutkin, 1973) and is involved in wound healing (Levenson, Crowley, Rettura, Dorinne, Gruber \& Seifter, 1976) and cell differentiation (De Luca, Maestri, Bonanni \& Nelson, 1972).

Our own clinical studies (Soukop, McAllister \& Calman, 1977) have shown that though the plasma levels of this vitamin are low in many patients the levels do not make a good index of nutritional status. Of greater significance, however, is that in a retrospective study (Soukop, McVie \& Calman, 1977) it was shown that the response rate to drug therapy was correlated with the plasma vitamin A level. A clinical trial is at present underway to evaluate, prospectively, the role of supplemental vitamin A in cancer chemotherapy.

Riboflavin. This vitamin has been extensively evaluated in cancer patients (Rivlin, 1973). Riboflavin antagonists have been used clinically as antitumour agents (Lane, 1971).

Trace metals. Trace metals are fundamental for many biological processes. In relation to cancer management, trace metals are important in monitoring therapy in the lymphomas (Mortazavi et al. 1972), the serum copper levels indicating whether the disease is active or not. From a nutritional support point of view, however, few replacement studies have been made. A recent short report (Cutler, Palmer \& Kontras, 1977) in which zinc replacement therapy was used, successfully, to improve mouth ulceration following chemotherapy does, however, indicate that it may have a role in patient management. It is of interest that appetite and taste were also stimulated during zinc therapy. 


\section{Nutritional support in cancer therapy - is it harmful?}

As enthusiasm grows for nutritional support it is essential that a critical look is taken at the potential harm which may result, apart from the expense involved (Burke \& Kark, 1977). There is no doubt that changes in the nutritional environment of host will alter the growth rate of tumours in experimental animals (Tannenbaum \& Silverstone, 1953), an increase in the energy intake increasing the incidence of tumours. Similarly changes in the nutritional status may alter the immunological response of the tumour-bearing animal. There is no clear evidence, however, that reversal of a nutritional problem would alter the immune response in an adverse way. At a clinical level the only evidence that would indicate a potentially harmful effect are the forced-feeding studies by Terepka \& Waterhouse (1956). It is essential to point out that the work referred to was performed without concomitant antitumour therapy. It is still possible therefore that nutritional support, while potentially stimulating tumour growth might be beneficial, if, at the same time, effective therapy was given. Indeed if the number of cells in division (the growth fraction) was increased then it might be more successful. Certainly in studies in which both nutritional support and antitumour therapy have been given together, no adverse effects have been noted. It is necessary, however, that great caution is taken in the design and evaluation of nutritional protocols in cancer patients, and that the tumour growth is also assessed.

The role of the dietician. No clinical study of nutritional aspects of cancer would be complete without close involvement of the dietician. The supervision of patient care and participation in research projects forms an essential, and ever increasing, part of their duties. The dietician is a crucial part of the cancer care team.

The potential for the future. In terms of nutritional support for the cancer patient, only the surface of this vast topic has been scratched. The potential benefit for patient care may be great of the areas which are of greatest interest at present are:

(r) The use of intravenous hyperalimentation in association with chemotherapy. The results referred to earlier are encouraging, and, with proper assessment and evaluation, it is hoped that controlled results will be available soon.

(2) A greater understanding of the nutritional and metabolic problems of cancer patients. The complex problems of cancer patients require further study both at an experimental level, and in the clinic. In the field of energy metabolisms, for example, there are many similarities between the catabolic patient, the tumourbearing animal, and the state of preserved organs. As the understanding of the fundamental problems increases so the potential for specific replacement therapy increases.

(3) Specific vitamin replacement therapy. Both with ascorbic acid and vitamin A there is a clear hope that specific replacement might increase the response rates to chemotherapeutic agents. The mechanisms of these vitamins require further study.

(4) Trace metals. The importance of these metals in biological systems is well known. Their role in cancer therapy, however, requires much more evaluation. However, the clues that exist suggest that the potential may be great. 
(5) Drug metabolism and nutrition. There is now very good evidence that the nutritional status of patients modifies the manner in which drugs are metabolized. Pharmacological studies with chemotherapeutic agents in different nutritional states are urgently needed. Nutritional modification in such patients may modify drug effectiveness.

(6) Nutrition supplementation of the normal diet. Many of these dietary products are being used indiscriminately in cancer patients. Controlled trials should be carried out to evaluate the advantages and disadvantages of such techniques.

\section{REFERENCES}

Anonymous (1976). Trends biochem. Sci. 1, 126.

Basu, T. K., Dickerson, J. W. T., Raven, R. W. \& Williams, D. C. (1974). Int. F. Vitam. nutr. Res. 44, 53 .

Bounous, G., Gentile, J. M. \& Hugon, J. (1971). Can. F. Surg. 14, 312.

Burke, M. \& Kark, A. E. (1977). Lancet i, 999.

Calman, K. C. (1976). In The Role of Elemental Nutrition, p. 30 [I. D. A. Johnston, editor]. Tunbridge Wells: M. C. S. Consultants.

Cameron, E. \& Pauling, L. (1976). Proc. natn. Acad. Sci. U.S.A. 73, 3685.

Cohen, M. H. \& Carbone, P. P. (1972). F. natn. Cancer Inst. 48, 921.

Copeland, E. M., Souchon, E. A., MacFadyen, B. V., Rapp, M. A. \& Dudrick, S. J. (1977). Cancer, N.Y. 39, 609.

Cutler, E. A., Palmer, J. \& Kontras, S. B. (1977). New Engl. J. Med. 297, 68.

De Luca, L., Maestri, N., Bonanni, F. \& Nelson, D. (1972). Cancer, N.Y. 30, I 326.

DeWys, W. D. \& Walters, K. (1975). Cancer, N.Y. 36, 1888.

Goodlad, G. A. \& Raymond, M. J. (1973). Eur. Y. Cancer 9, 139.

Jurin, M. \& Tannock, I. F. (1972). Immunology 23, 283.

Lane, M. (1971). Proc. Am. Ass. Cancer Res. 12, 85.

Lanzotti, V. J., Copeland, E. M. 3rd, George, S. L., Dudrick, S. J. \& Samuels, M. L. (1975). Cancer Chemother. Rep. 59, 437.

Levenson, S. M., Crowley, L. V., Rettura, R., Dorinne, K., Gruber, B. A. \& Seifter, E. (1976). In Metabolism and the Response to Injury, p. 253 [A. W. Wilkinson and D. P. Cuthbertson, editors]. Tunbridge Wells: Pitman Medical.

Meltzer, M. S. \& Cohen, B. E. (1974). F. natn. Cancer Inst. 53, 585.

Mortazavi, S. H., Bani-Hashemi, A., Mozafari, M. \& Raffie, A. (1972). Cancer, N.Y. $29,1193$.

Newberne, P. M. \& Rogers, A. E. (1973). F. natn. Cancer Inst. 50, 439.

Okuda, H., Ikegami, H. \& Fujii, S. (1972). Gann 63, 605.

Pareira, M. D., Conrad, J. M., Hicks, W. \& Elman, R. (1955). Cancer, N. Y. 8, 803.

Pories, W. J., Mansour, E. G. \& Strain, W. H. (1972). Ann. N.Y. Acad. Sci. 199, 265.

Prutkin, L. (1973). Cancer Res. 33, 128.

Rivlin, R. S. (1973). Cancer Res. 33, 1977.

Samuels, M. L., Lanzotti, V. J., Hologe, P. Y., Boyle, L. E., Smith, T. L. \& Johnson, D. E. (1976). Cancer Treat. Rev. 3, 185.

Seifter, E., Rettura, G., Demetrion, A. A. \& Levenson, S. (1976). F. natn. Cancer Inst. 57, 355.

Soukop, M., McAllister, R. A. \& Calman, K. C. (1977). Scott. Med. J. 22, 189.

Soukop, M., McVie, J. G. \& Calman, K. C. (1977). Proc. Int. Congress of Chemotherapy, Zurich. (In the Press).

Stitch, H. F., Karim, J., Koropatnick, J. \& Lo, L. (1976). Nature, Lond. $260,722$.

Sydner, K. L., Allen, C. \& Flesher, J. W. (1972). F. natn. Inst. 48, 92 I.

Tannenbaum, A. \& Silverstone, H. (1953). Adv. Cancer Res. I, 45 I.

Terepka, A. R. \& Waterhouse, C. (1956). Am. F. Med. $20,225$.

Theoglides, A. (1972). Cancer, N.Y. 29, 484.

Zannoni, V. G. \& Rikans, L. E. (1976). Trends biochem. Sci 1, 126.

\section{Printed in Great Britain}

